\title{
Devising a cost-effective method to improve the specificity and sensitivity of malaria detection
}

Sanders Sebastian ( $\nabla$ sandersvs@hotmail.com )

Dorevtch Pathology https://orcid.org/0000-0003-1367-5587

\section{Short Report}

Keywords: malaria parasites, quantitative buffy coat, malaria detection

Posted Date: November 15th, 2021

DOI: https://doi.org/10.21203/rs.3.rs-1056804/v1

License: (9) This work is licensed under a Creative Commons Attribution 4.0 International License. Read Full License 


\section{Abstract \\ Background:}

It has always been a challenge to increase the concentration of malaria parasites in blood without compromising on specificity. The conventional thick smear method is a good sensitive tool. But the disadvantage is that a lot of expertise and experience is needed to perform this. A pilot study was undertaken to improvise the quantitative buffy coat (QBC) method so that the advantages of both thick and thin smears could be combined.

\section{Methods:}

Used QBC capillary tubes were washed off the blood and the dye and thoroughly cleaned and dried. The floats were preserved. Blood samples of ten malaria positive patients collected already in EDTA bottles were drawn onto the washed and dried QBC capillaries. The float was re-inserted and centrifugation done as recommended by the QBC manufacturers. The capillary tubes were broken with the aid of a diamond pencil at the area were parasites infested cells were most likely to be seen ie just below the buffy coat area. Smears were made onto a slide with this material and stained using the conventional Romanowsky method of staining. Ordinary microscopy was carried out and parasitaemia were quantified as number per oil immersion field and the results were compared with the recorded thin film reports. Five known negative controls were treated similarly.

\section{Results:}

Parasites' number was found to be on an average ten times higher per oil immersion field compared to the conventional methods. Negative controls yielded valid outcomes.

\section{Conclusion:}

This preliminary study promises to be a useful tool in screening for malaria in endemic areas where resources are limited. It also adds to the specificity compared with the original QBC method. Larger study involving more samples is required to further validate the results. Any easy method (preferably automated) to cut the capillary tube thereby eliminating the risk of infection to the operator would help a long way in making the method more user friendly.

\section{Introduction}

It has always been a challenge to increase the concentration of malaria parasites in blood without compromising on specificity. The conventional thick smear method is a good sensitive tool. But the disadvantage is that a lot of expertise and experience is needed to perform this. 
Alternative methods to concentrate the parasites started as early as the beginning of the 20th century. These methods were based on sedimentation (Bass and Johns, 1916 cited in Worth, 1964, p.71) or differential centrifugation (Ferrebee and Geiman, 1946 cited in Worth,1964, p71). Bennett (1962, cited in Worth, 1964 p.71) found that centrifugation concentrated haemoparasites just below and above the buffy coat. These efforts underwent a revolution with the advent of quantitative buffy coat analysis (Wardlaw and Levine, 1983, p.617). QBC was originally designed "to provide a rapid means of performing a complete blood cell count in a physician's office."

The main difference this method had with the earlier efforts was that it physically expanded and separated the buffy coat into three distinct layers of granulocytes, non-granulocytes and platelets in a capillary tube. A cylindrical plastic 'float' is inserted into the capillary tube here. This has a specific gravity in between that of plasma and RBCs. So when the blood is centrifuged the float settles in the buffy coat area. The latter expands around the float owing to the limited availability of room inside the capillary. The capillary tube is pre-coated with a fluorescent dye (acridine orange) which is taken up by the nucleoproteins. Micrometric measurements of the separated regions in fluorescent light enable the quantification of the different cell layers. This same group of researchers observed that blood borne parasites like malarial trophozoites were detectable within the expanded area.

Later, Spielman et al (1988, p.337) demonstrated that "red blood cells infected with diverse stages of Plasmodium falciparum and $P$. vivax are lighter than non-infected cells and somewhat heavier than granulocytes; thus, they can readily be detected by direct inspection of UV-illuminated tubes". They found that centrifugation using the QBC capillary tubes for malaria screening enhanced the detection rates by at least eight times compared to the conventional thin film method, considered the gold standard for malaria detection. However, the authors admit that the method could not differentiate between the species of malaria. The other disadvantage is that a microscope with fluorescent attachment is essential here, a tall order in poor countries where malaria is prevalent. "When the QBC system is taken to the field away from the air-conditioning and reliable power supplies to the humid and impoverished reality of the malarious tropics, it does not do so well. Confusing fluorescent material precipitates inside the tubes if they are exposed to a very humid condition for more than a few days" (Baird, Purnomo and Jones, 1988 cited in Anon. 1992, p.1022)

Aim:

To devise a technique to eliminate the above disadvantages of the promising QBC method of malaria detection.

\section{Materials And Methods}

Used QBC capillary tubes were washed off the blood and the dye and thoroughly cleaned and dried. The floats were preserved. Blood samples of ten malaria positive patients collected already in EDTA bottles were drawn onto the washed and dried QBC capillaries. The float was re-inserted and centrifugation done as recommended by the QBC manufacturers. The capillary tubes were broken with the aid of a diamond 
pencil at the area were parasites infested cells were most likely to be seen ie just below the buffy coat area. Smears were made onto a slide with this material and stained using the conventional Romanowsky method of staining. Ordinary microscopy was carried out and parasitaemia were quantified as number per oil immersion field and the results were compared with the recorded thin film reports. Five known negative controls were treated similarly.

\section{Results}

Parasites' number was found to be on an average ten times higher per oil immersion field compared to the conventional methods (See photograph below). Negative controls yielded valid outcomes.

\section{Conclusion}

This preliminary study promises to be a useful tool in screening for malaria in endemic areas where resources are limited. It also adds to the specificity compared with the original QBC method. Larger study involving more samples is required to further validate the results. Any easy method (preferably automated) to cut the capillary tube thereby eliminating the risk of infection to the operator would help a long way in making the method more user friendly.

\section{Declarations}

- Ethics approval and consent to participate: Not applicable

- Consent for publication: Not applicable

- Availability of data and materials: Photographs

- Competing interests: None

- $\quad$ Funding: None

- Authors' contributions: Sanders Sebastian conceptualised and executed this innovation and

- wrote the article.

- Acknowledgements: Not applicable

- Authors' information (optional): Sanders Sebastian, Biochemist/ Medl Scientist/ Clinical

- Researcher : https://www.linkedin.com/feed/

\section{References}

1. Anon. (1988), 'QBC malaria diagnosis', The Lancet, 339, April, pp. 1022.

2. Spielman, A., Perkone, J.B., Teklehaimanot, A., Balcha, F., Wardlaw, S.C., and Levine, R.A. (1988), 'Malaria diagnosis by direct observation of centrifuged samples of blood', The American Journal of Tropical Medicine and Hygiene, 39 (4), April, pp. 337-342 
3. Wardlaw, S.C. and Levine, R.A. (1983), 'Quantitative buffy coat analysis: A new laboratory tool functioning as a screening complete blood cell count', The Journal of the American Medical Association, 249 (5), February, pp. 617-620.

4. Worth, R. M. (1964) 'Heparinised capillary tube as an epidemiological tool: concentration of blood parasites by centrifugation', The American Journal of Hygiene, 80, February, pp. 70

\section{Figures}
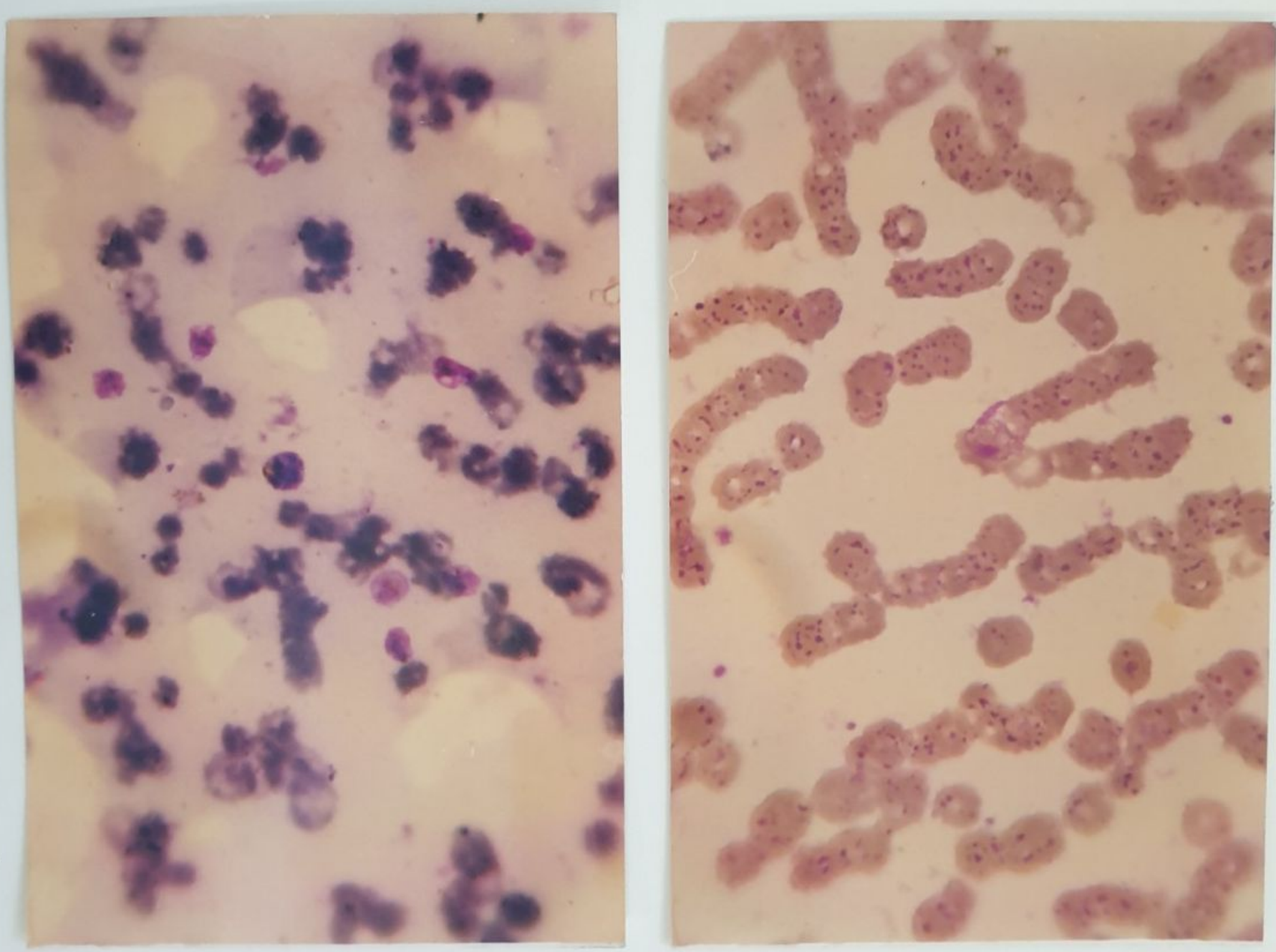


\section{Figure 1}

The photo on the left is that of the modified technique and on the right is of the traditional thin smear of the same sample. 